- 28 February that it would not renew its contract, because Elsevier was demanding too high a price for the deal. UC's latest subscription with the publisher expired on 31 December, and researchers' access to Elsevier journals had been extended while negotiations continued.

UC pays about US\$11 million a year to Elsevier in subscription fees, and the publisher wanted to increase the cost by about $80 \%$, according to the institution's calculations, said Jeffrey MacKie-Mason, co-chair of the UC negotiating team, in an interview with Berkeley News, a website maintained by the university.

In a statement to Nature's news team, Elsevier called UC's decision "disappointing", and said that it had offered a model in which researchers could publish for free or as open access, and a path to reduce the costs for each UC campus.

In the past few years, stand-offs between academic publishers and institutes have increased in Europe, where several publishers have struck read-and-publish deals with university consortia. Other US institutions, including Florida State University in Tallahassee, have cancelled major subscription deals with Elsevier over concerns about costs, but have continued to pay for access to a small subset of journals.

Researchers in European countries, including Sweden and Germany, have been without access to new papers in Elsevier journals for months, while national library consortia try to negotiate deals.

\section{SHORT-TERM PAIN}

Some UC researchers welcomed the institution's decision. "I'm ecstatic," says Jeffrey Ross-Ibarra, a plant geneticist at the University of California, Davis. He predicts some "short-term pain" as researchers determine how to access articles without a subscription.

Jay Keasling, a chemical engineer at UC Berkeley, has mixed feelings about the situation. Many students and scientists won't have access to publications, he says. But "Elsevier is a bit of a monopoly and I totally get where the university is coming from," he says. "I wish they could have gotten to some point of agreement." Keasling, co-editor-in-chief of an Elsevier journal, Metabolic Engineering, also worries that the break will affect the quality of the publisher's titles.

UC academics will still have access to much of Elsevier's back catalogue and will lose access only to articles published in Elsevier journals since the expiry of the institution's licence, because of contract clauses that cover 'post-termination access'.

Elsevier, headquartered in Amsterdam, publishes nearly 3,000 journals, which issue about 400,000 papers a year. UC has 10 campuses and says that $18 \%$ of its researchers' published studies are in Elsevier journals. .

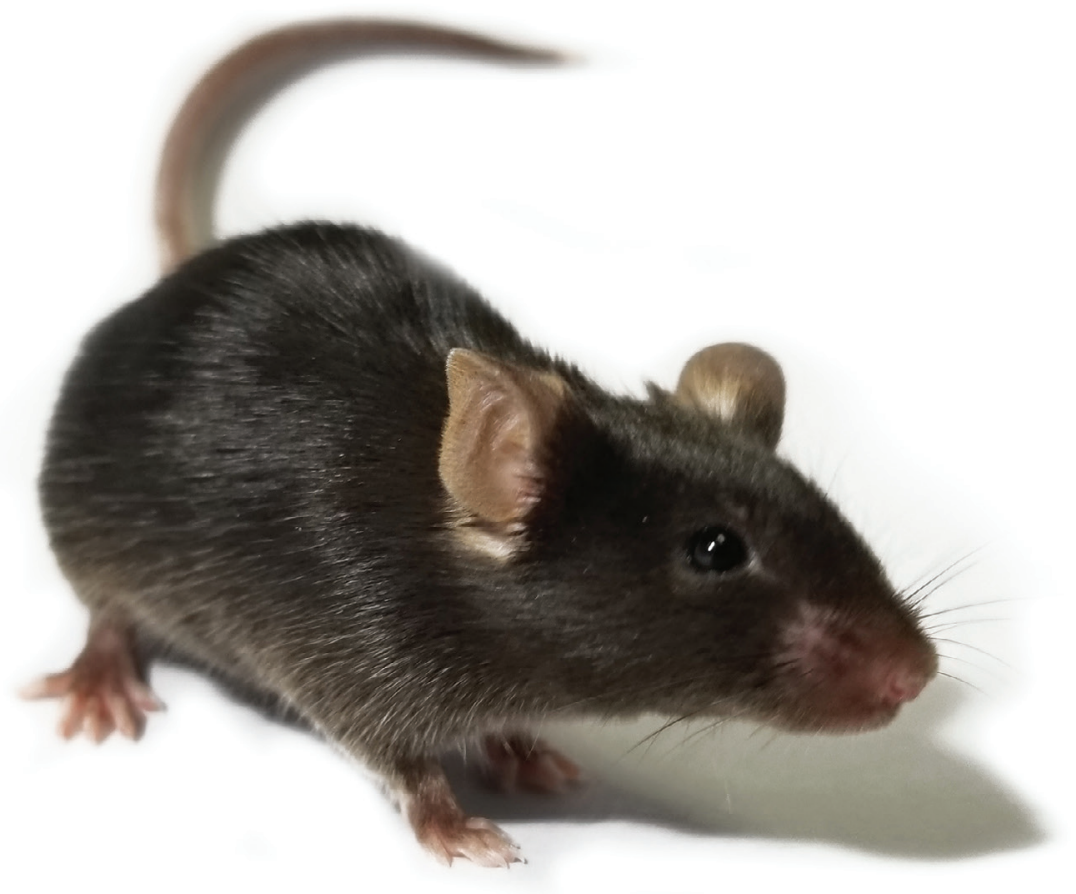

Engineered mice were able to see infrared light as visible light.

\section{ENHANCEMENT}

\section{'Super-mice' see in the dark}

\section{BY MATTHEW WARREN}

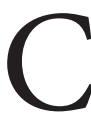

ue the super-mouse. Scientists have engineered mice that can see infrared light normally invisible to mammals including humans.

To do so, they injected into the rodents' eyes nanoparticles that convert infrared light into visible wavelengths (Y. Ma et al. Cell http:// doi.org/gfv5c8; 2019). Infrared light has wavelengths slightly longer than red light between 700 nanometres and 1 millimetre.

Tian Xue, a neuroscientist at the University of Science and Technology of China in Hefei, and his colleagues developed nanoparticles that transform infrared wavelengths into visible light. The nanoparticles absorb photons at wavelengths of around 980 nanometres and emit them at shorter wavelengths, around 535 nanometres, corresponding to green light.

Xue's team attached the nanoparticles to proteins that bind to photoreceptors - the cells in the eye that convert light into electrical impulses - and then injected them into the mice.

The researchers showed that the nanoparticles successfully attached to the photoreceptors, which in turn responded to infrared light by producing electrical signals and activating the visual-processing areas of the brain.

\section{NIGHT-VISION GAMES}

The team conducted experiments to show that the mice did actually detect and respond to infrared light.

In one test, they gave mice the choice between a dark box and a box 'illuminated' with infrared light. Normally, mice - which are nocturnal - will seek out the safety of a darker box. The ordinary mice showed no preference between the two boxes because they couldn't see the infrared light. But the modified mice favoured the dark box.

In another experiment, the team taught both types of mouse to associate green light with an electric shock. The modified mice also froze in fear when an infrared light was turned on.

Finally, the researchers placed the rodents in a water maze that had two arms illuminated by different light patterns, only one of which led to a hidden, dry refuge. The modified mice chose the correct arm of the maze according to the light pattern, regardless of whether the patterns were displayed in visible or infrared light. 
"It's sometimes a little bit creepy," says Xue. "You show different patterns to the mouse which you cannot see - to you, it's just an empty screen. But the mouse can choose it correctly."

Other groups have also sought to give rodents infrared vision. Eric Thomson, a neuroscientist at Duke University in Durham, North Carolina, developed a system that allowed rats to detect infrared light through four sensors connected directly to the brain (K. Hartmann et al. J. Neurosci. 36, $2406-2424 ; 2016)$. But the small number of sensors only provided enough visual information for the rats to find the location of a light, says Thomson. "What is really exciting here is that they actually showed that they got real image information."

Xue says that his technique could have several applications, including giving people "super-vision". Seeing infrared light could help people to see at night, by enabling them to detect infrared wavelengths emitted by, or reflected off, people and objects in the environment. This could be useful for military and security operations, for example.

The team also hopes to adapt the nanoparticles to carry drugs for later release in the eye. But there are several hurdles, including safety concerns, before any use in humans can be tested. For example, the team's nanoparticles contained heavy metals and regulators would be unlikely to approve them for use in humans, Xue says, so the team is developing organic versions.

But not everyone thinks that this technique could be used to augment human vision. The human visual system has evolved over millions of years to be sensitive to a highly specific part of the electromagnetic system, says Glen Jeffery, a visual neuroscientist at University College London, and the retina is not used to seeing infrared. It's uncertain how people would interpret the image: the environment would appear a lot brighter, for example, and the images could be overwhelming.

"I am the last person in the world who would want to see infrared," says Jeffery. .

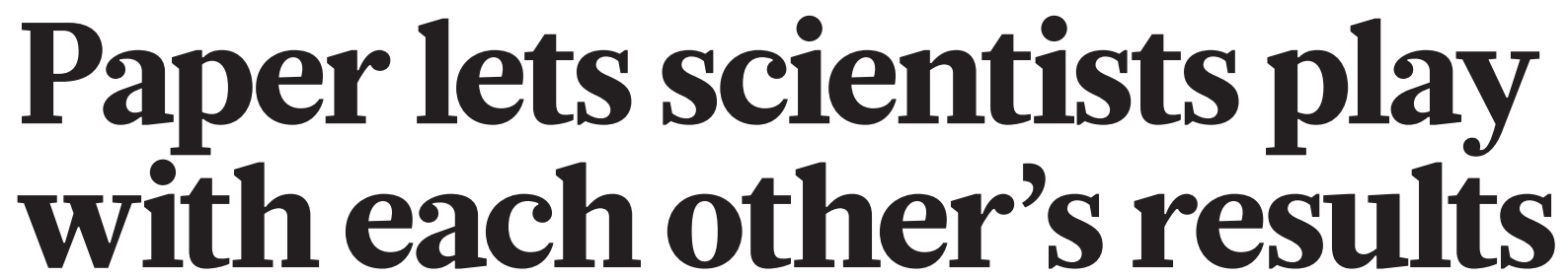

\section{Online journal eLife creates paper that lets readers change the code underlying figures.}

\section{BY JEFFREY M. PERKEL}

$\mathrm{T}$ The online journal eLife has taken a significant step towards a future in which its papers are much more than just static pages. Readers of the journal's first "computationally reproducible" article can change the code underlying figures to better understand, validate or build on the work. For example, they can rerun the code to see what a figure looks like without outliers, or represented as a different type of plot (see go.nature.com/2c3a9fq).

"What eLife is doing is making this commitment to upgrading the research article so that it is not just the written word, but it is this multifaceted communication medium," says Lorena
Barba, a mechanical and aerospace engineer and reproducibility specialist at the George Washington University in Washington DC.

The article, which eLife first published in its conventional format last year (L. M. Lewis et al. eLife 7, e30274; 2018), is a prototype of technologies the journal now plans to scale up, says Giuliano Maciocci, head of product and user experience at eLife in Cambridge, UK. Authors who would like to exploit similar features can contact the journal for consideration, he adds.

In future, such articles could make it easier for researchers to reuse each other's code. Users can't upload their own data and add them to figures, but Maciocci says that the plan is for them eventually to be able to download such articles

\section{COMPUTATIONAL REPRODUCIBILITY}

Readers of the first computationally reproducible article published by the journal eLife can tweak the underlying code to change the figures. In this case, the authors' original figure (left) was altered to change its chart type and coloration.
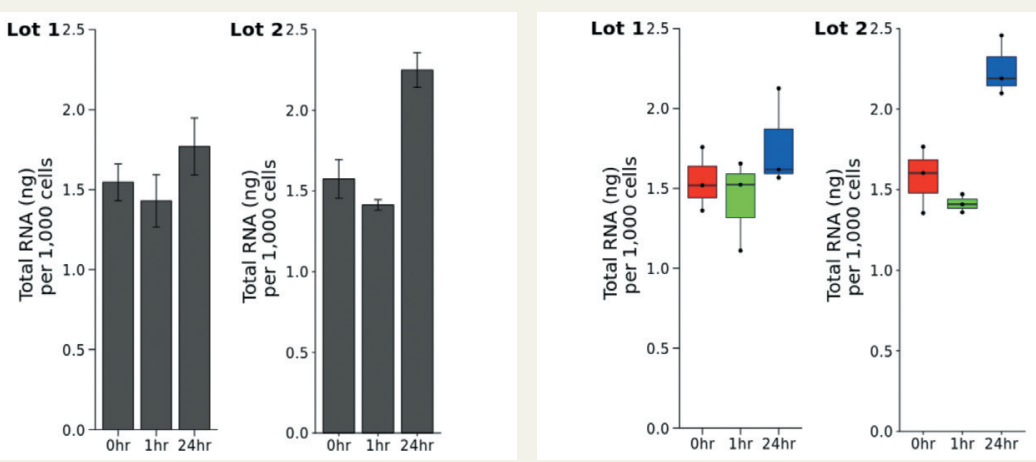

and run them to, for instance, analyse their own findings using the authors' code. Reusing such software is often surprising difficult, involving confusing sets of interdependent tools, each of which must be downloaded and installed. But eLife's proof-of-concept publication allows users to view and execute code in the body of the article itself, with no installation required.

The ability to make reproducible documents is not new, notes Titus Brown, a bioinformatician at the University of California, Davis. Researchers can do it themselves by combining tools such as Jupyter Notebook, an interactive lab notebook, with the cloud-based software Binder that allows others to execute the code. "What's been lacking is the integration with the publisher side of things," says Brown.

Some journals, including F1000Research, GigaScience and titles from Cell Press, already allow authors to embed executable 'compute capsules' from the cloud-based platform Code Ocean in their articles, with the code and execution environment rendered as an interactive widget. And in August 2018, Nature Methods, Nature Biotechnology and Nature Machine Intelligence launched an ongoing pilot programme with Code Ocean to use the company's compute capsules for peer review. But in the eLife article, the code is a native part of the article itself.

The eLife paper describes an attempt to replicate a 2012 paper about how a gene that is often mutated in cancer cells impacts the expression of other genes (C. Y. Lin et al. Cell 151, 56-67; 2012). The study was conducted as part of the Reproducibility Project: Cancer Biology, 